\title{
Holographic Microwave Induced Imaging System: A Numerical Study
}

\author{
Lulu Wang ${ }^{1,2, *}$ \\ ${ }^{1}$ School of Instrument Science and Opto-electronics Engineering, Hefei University of Technology, China \\ ${ }^{2}$ Institute of Biomedical Technologies, Auckland University of Technology, New Zealand \\ ${ }^{*}$ Corresponding author
}

\begin{abstract}
This paper presents a holographic microwave induced (HMI) imaging method based on microwave imaging and ultrasound imaging techniques to detect breast lesion. A numerical system, including a breast model and an HMI measurement model, is developed to investigate the feasibility of breast tumor detection. Results show that the small breast lesion can be clearly detected in the HMI images. The proposed framework has the potential to become a useful tool for breast lesion detection.
\end{abstract}

Keywords—microwave imaging; elasticity imaging; holographic; microwave antenna; breast cancer

\section{INTRODUCTION}

Microwave imaging has been proposed as an alternative or additional approach to the conventional mammography for the early diagnosis of breast cancer [1]. MI-based approaches have not been extensively studied in clinical settings due to several limitations include long scanning time and limited image resolution [2]. To solve these challenges, develop a high dynamic MI technique has attacked many researchers' interests over the past two decades [3-5]. Harmonic motion imaging method has been proposed to detect breast lesion based on the elastic parameters contrast between the healthy and abnormal tissues [6]. In this method, the acoustic radiation force of focused ultrasound is applied to generate a locally oscillatory displacement inside the soft tissue. The produced pressure variation in normal tissue is different from that in abnormal tissue due to the dielectric properties contrast between the healthy and abnormal tissues. The authors recently developed a holographic microwave imaging technique for breast lesion detection [7], which has the potential to produce a highresolution image. The image quality is improved significantly by using a spiral antenna array [8].

This paper aims to investigate the feasibility of using holographic microwave induced (HMI) imaging that combines the benefits of harmonic motion imaging and holographic microwave imaging techniques to identify breast tumors based on monitoring the electrical and mechanical properties of the breast model. A computer simulation system includes a breast model and signal processing model is developed to demonstrate the working principle of HMI. Various simulation experiments are conducted to evaluate the effectiveness and performance of the proposed HMI method.

\section{THEORY}

Figure 1 shows the schematic diagram of HMI. The system consists of a microwave generator, a microwave antenna array includes a transmitter and several receivers, a focused ultrasound probe, and a breast model. The microwave antenna array is placed in far field distance to the breast model.

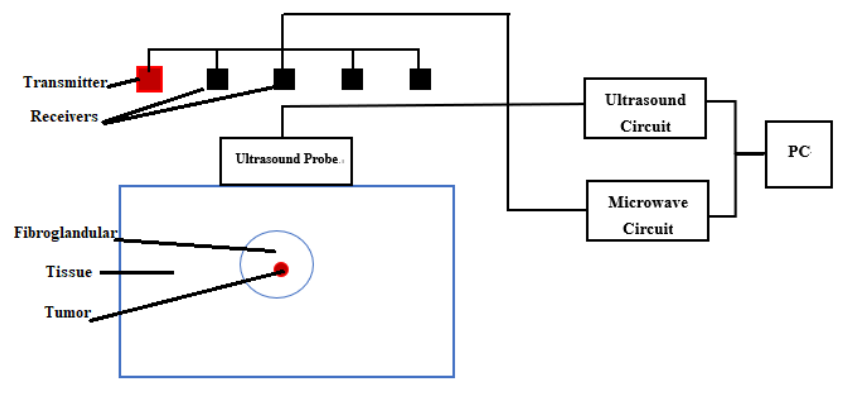

FIGURE I. ILLUSTRATION OF THE HMI SYSTEM

During data collection, the microwave generator generates microwave signals to the breast through the microwave transmitter, at the same time, the ultrasound probe induces vibration inside the tissue, and the backscattered signals from the target region are recorded by each microwave receiver. The received signals contain phase and amplitude information due to holographic technique and vibration. These signals depend on the dielectric properties, volume and maximum displacement of the vibrating region. The scattered energy is changed if the vibrating region contains a different material from the background. This response is related to both the mechanical and electrical parameters of the tissue. Thus, more useful information can be obtained than the microwave or elasticity imaging.

Define breast intensity as [7]:

$$
I(\vec{s})=\left(\frac{k_{0}^{2}}{4 \pi}\right)^{2}\left|\varepsilon_{r}(\vec{s})-\varepsilon_{0}\right|^{2} \overrightarrow{E_{T}}(\vec{s}) \cdot \overrightarrow{E_{T}^{*}}(\vec{s})
$$

Where $k_{0}$ denotes the wavenumber of free-space, $\Delta x_{\mu}(\overrightarrow{\mathrm{s}})=$, $\varepsilon_{r}$ is the relative permittivity of object, $\varepsilon_{0}$ is the relative permittivity of free-space, $\overrightarrow{E_{T}}=\vec{E}_{\text {inc }}+\vec{E}_{\text {scat }}, \overrightarrow{E_{T}}, \vec{E}_{\text {inc }}$ and $\vec{E}_{\text {scat }}$ are the total electric field, incident electric field and scattered electric field, respectively.

Acoustic attenuation is mainly caused by absorption, and the applied force to the target object can be represented as [9]:

$$
F=\frac{\alpha P_{0}^{2}}{\rho c^{2}} \cos ^{2}(2 \pi f t)
$$


Where $\alpha$ denotes the absorption constant, $c$ denotes the velocity of ultrasound in the tissue, $\rho$ denotes the tissue density, $f$ is the operating frequency of the ultrasound sensor, $\mathrm{P}_{0}$ denotes the peak pressure at transducer $\mathrm{P}_{0}=\sqrt{\frac{2 \rho c P}{\pi /\left(a^{2}-b^{2}\right)}}$, $\mathrm{P}$ is the ultrasound probe power, $a$ and $b$ are the board and narrow band of the ultrasound sensor, respectively.

The displacement of the local tissue can be written as [10]:

$$
X(t)=X_{0} \cos (2 \pi f t+\phi)
$$

Where $\mathrm{X}_{0}$ denotes the maximum displacement, which depends on the mechanical properties of the local tissue and the intensity of the applied ultrasound force. $\phi$ denotes the phase change, which depends on the total path length.

If the target breast lesion is relatively small, then the polarization density inside the lesion can be obtained [11]:

$$
\vec{P}=\frac{3 \varepsilon_{b}\left(\varepsilon_{r}-\varepsilon_{b}\right)}{\varepsilon_{r}+2 \varepsilon_{b}} \varepsilon_{0} \vec{E}_{\text {inc }}
$$

Where $\varepsilon_{b}$ denotes the relative permittivity of the surrounding medium. $\vec{E}_{\text {inc }}$ is the incident field on the breast lesion.

The microwave transmitter transmits microwave signals to the breast model:

$$
\vec{E}_{\text {inc }}=\left(-\frac{j k_{0}}{2 \pi^{2}}\right) \vec{E}_{0}\left(\frac{e^{-j k_{0} \vec{R}} \overline{T_{x_{m}}}}{\vec{R}_{\overline{x_{m}}}}\right) A_{N} B_{B} h(\theta, \emptyset) \overrightarrow{P O}(\theta, \emptyset)
$$

Where $\overrightarrow{R_{\vec{x}}}$ denotes the distance vector from the breast to the transmitter located at $\overrightarrow{T_{x}}, \vec{E}_{0}$ is the wave amplitude of TE10 mode, $A_{N}$ and $B_{B}$ are the narrow and broad aperture dimensions of antenna aperture, respectively. $h(\theta, \varnothing)$ is radiation pattern, and $\overrightarrow{P O}(\theta, \varnothing)$ is the polarization vector, $k_{0}$ denotes the wavenumber of free-space. $k_{0}=2 \pi f_{1} t / c_{1}, f_{1}$ denotes the operating frequency of microwave antenna, $c_{1}$ denotes the speed of light.



FIGURE II. MICROWAVE ANTENNA PAIR
Referring to Figure 2, the scattered signals measured by the microwave receiver located at $\vec{r}_{l}$ can be represented as:

$$
\begin{gathered}
\vec{E}_{\text {scat }}\left(\overrightarrow{r_{m}}\right)=\left(\frac{k_{0}^{2}}{4 \pi}\right) \int_{V}\left|\varepsilon_{r}(\vec{s})-\varepsilon_{0}\right|\left\{a \vec{E}_{T}(\vec{s})\right. \\
\left.+\left(b \vec{E}_{T}(\vec{s}) \cdot \widehat{R_{n}}\right) \widehat{R_{n}}\right\} G\left(\overrightarrow{r_{m}}, \vec{s}\right) d V
\end{gathered}
$$

Where $G$ denotes the green function.

The total scattered signals from any two receivers located at $\vec{r}_{l}$ and $\vec{r}_{j}$ can be presented as [8]:

$$
\Delta \vec{E}_{\text {scat }}\left(\vec{r}_{l}, \vec{r}_{j}\right)=<\vec{E}_{\text {scat }}\left(\vec{r}_{l}\right) \cdot \vec{E}_{\text {scat }}^{*}\left(\vec{r}_{J}\right)>
$$

Where $\vec{E}_{\text {scat }}$ and $\vec{E}_{\text {scat }}^{*}$ are the scattered field and the conjugate complex of the scattered field, respectively. The scattered filed contains phase and amplitude information.

Referring to Figure 2, the breast image can be obtained by:

$$
\tilde{I}=\iint \Delta \vec{E}_{s c a t}\left(u_{i j}, v_{i j}\right) \mathrm{e}^{-j 2 \pi\left(u_{i j} l+v_{i j} m\right)} d u d v
$$

Where $l=\sin \theta \cos \phi, m=\sin \theta \sin \phi, u_{i j}=\left(\overrightarrow{x_{j}}-\overrightarrow{x_{l}}\right) /$ $\lambda_{b}$, and $v_{i j}=\left(\overrightarrow{y_{j}}-\overrightarrow{y_{l}}\right) / \lambda_{b} . \theta$ and $\phi$ are the receiver angle to the $\mathrm{z}$ direction and the angle to the $\mathrm{x}-\mathrm{y}$ direction, respectively. $\lambda_{b}$ denotes the wavelength of microwave signal.

\section{NUMERICAL EXPERIMENTS}

A numerical system was developed using MATLAB to validate the proposed HMI method for breast tumor detection. The system made of a microwave sensor array, a focused ultrasound transducer, and a breast model. The model $\left(100 \times 100 \times 50 \mathrm{~mm}^{3}\right)$ was made of skin $(2 \mathrm{~mm})$, fat, gland, and two spherical-shaped tumors (4mm in diameter), and it contained $256 \times 256 \times 55$ elements (Figure 3 ). The antenna array made of one transmitter and 15 receivers and a small openended waveguide antenna was simulated as both transmitter and receiver. The ultrasound probe and transmitter were placed next to the breast model, while receivers were randomly located in a 2D array plane $\left(300 \times 300 \mathrm{~mm}^{2}\right)$ and they were in far-field distance from the breast model.

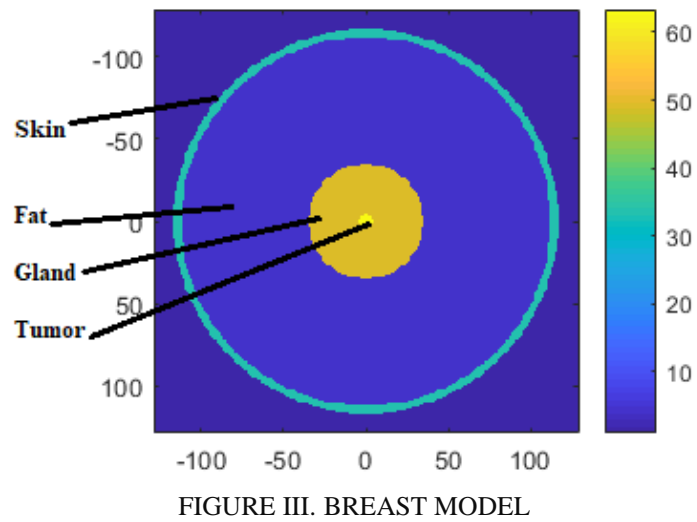


Table 1 shows the operating frequency and setup parameters for the simulation system [12]. Table 2 shows the attenuation and the velocity of the ultrasound [6].

\section{TABLE I. SIMULATION PARAMETERS [12]}

\begin{tabular}{|l|l|}
\hline The frequency of microwave $(\mathrm{GHz})$ & 5,6 \\
\hline The frequency of ultrasound $(\mathrm{MHz})$ & 3 \\
\hline Microwave antenna width & $7.5 \mathrm{~mm}$ \\
\hline Microwave antenna length & $15 \mathrm{~mm}$ \\
\hline The relative permittivity of the gland & $60.537-0.93424 \mathrm{j}$ \\
\hline The relative permittivity of fat & $5.475-0.75 \mathrm{j}$ \\
\hline The relative permittivity of skin & $43.635-0.76519 \mathrm{j}$ \\
\hline The relative permittivity of free-space & 1 \\
\hline The relative permittivity of tumor & $62.603-0.61063 \mathrm{j}$ \\
\hline
\end{tabular}

TABLE II. ULTRASOUND PARAMETERS [6]

\begin{tabular}{|c|c|c|}
\hline & $\begin{array}{c}\text { Attenuation } \\
(\mathrm{dB} / \mathrm{cm} / \mathrm{MHz})\end{array}$ & $\begin{array}{c}\text { Velocity } \\
(\mathrm{m} / \mathrm{s})\end{array}$ \\
\hline Fat & 0.34 & 1479 \\
\hline Tumor & 0.79 & 1550 \\
\hline Gland & 1.5 & 1553 \\
\hline
\end{tabular}

\section{Simulation RESUlts}

Figure 4 shows the reconstructed images of the breast model when the microwave frequency is $5 \mathrm{GHz}$. It can be seen that the target small breast lesion only can be found in the real part of the reconstructed image.

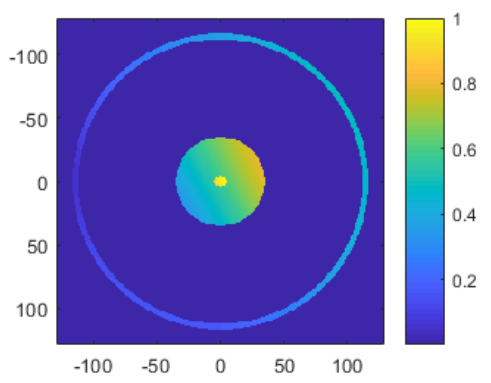

(a)

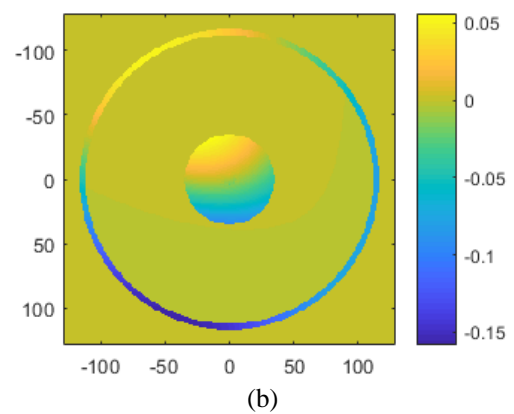

FIGURE IV. RECONSTRUCTED IMAGE OF BREAST (A) REAL-PART; (B) IMAGINARY-PART

Figure 5 shows the reconstructed images of the breast model when the microwave frequency is $6 \mathrm{GHz}$. It can be seen that the target small breast lesion can be found in both real-part and imagery-part of the reconstructed images.

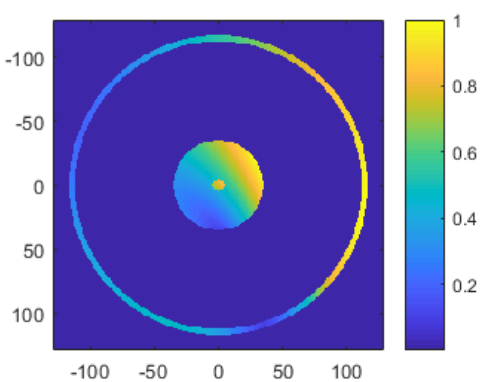

(a)

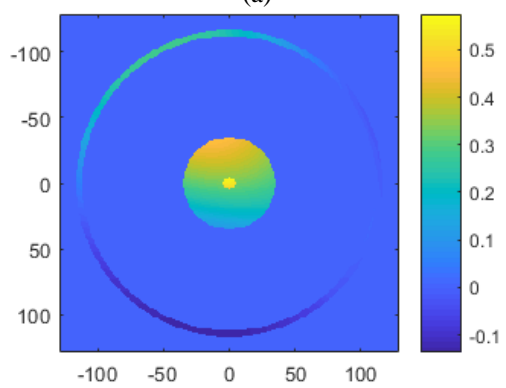

(b)

FIGURE V. RECONSTRUCTED IMAGE OF BREAST (A) REAL-PART; (B) IMAGINARY-PART

It can be seen that small breast lesion can be clearly detected when then the microwave frequency is $6 \mathrm{GHz}$. However, only the real part of the reconstructed image can identify the breast lesion when the microwave frequency is $5 \mathrm{GHz}$. Therefore, $6 \mathrm{GHz}$ can be selected as microwave operation frequency for the measurement system.

\section{CONCLUSIONS}

This paper demonstrated an imaging method namely HMI for small breast lesion detection. A numerical computer system consists of a simplified breast model, and the measurement model was developed to validate the proposed method. Several numerical experiments were conducted to confirm that the proposed method has the potential for breast lesion detection in the future.

\section{ACKNOWLEDGMENT}

This research was financially supported by the National Natural Science Foundation of China (Grant No. 61701159), the Natural Science Foundation of Anhui Province (Grant No. 101413246, JZ2017AKZR0129), the Fundamental Research Funds for the Central Universities (JZ2018HGTB0236), the Foundation for Oversea Master Project from Ministry of Education, China (Grant No. 2160311028), and the funding from Hefei University of Technology (JZ2018HGTB0236).

\section{REFERENCES}

[1] N. K. Nikolova, “Microwave imaging for breast cancer," IEEE Microwave Magazine, vol. 12, no. 7, pp.78-94, 2011.

[2] E. Porter, M. Coates, M. Popovi'c, "An early clinical study of timedomain microwave radar for breast health monitoring,” IEEE Trans. Biomed. Eng. vol. 63, pp.530-539, 2016.

[3] R. Chandra, H. Zhou, I. Balasingham, R.M. Narayanan, "On the opportunities and challenges in microwave medical sensing and imaging," IEEE Transactions on Biomedical Engineering, vol. 62, no.7, pp.16671682, 2015. 
[4] E. Porter, H. Bahrami, A. Santorelli, B. Gosselin, L. Rusch, M. Popovich, "A wearable microwave antenna array for time-domain breast tumor screening,” IEEE Trans. Med. Imaging, vol. 35, pp. 1501-1509, 2016.

[5] Klemm, M., Craddock, I., Leendertz, J., Preece, A. Experimental and clinical results of breast cancer detection using UWB microwave radar. Proceedings of the IEEE Antennas and Propagation Society International Symposium, San Diego, CA, USA, 2008, 1-4.

[6] Top, C. B., Gençer, N. G. "Harmonic motion microwave doppler imaging: a simulation study using a simple breast model.” IEEE Transactions on Medical Imaging, 2014, 33(2), 290.

[7] L. Wang, A.M. Al-Jumaily, R. Simpkin, "Imaging of 3-D dielectric objects using far-field holographic microwave imaging technique," Progress in Electromagnetics Research B, vol. 61, pp.135-147, 2014.

[8] L. Wang, A.M. Al-Jumaily, R. Simpkin, "Investigation of antenna array configurations using far-field holographic microwave imaging technique," Progress in Electromagnetics Research M, vol. 42, pp.1-11, 2015.

[9] K.R.Nightingale, M. L. Palmeri,R. W. Nightingale, G. E. Trahey, “On the feasibility of remote palpation using acoustic radiation force," J. Acous. Soc. Amer., 2001, 110, 625-634.

[10] M. Fatemi, J. F. Greenleaf, "Vibro-acoustography: An imaging modality based on ultrasound-stimulated acoustic emission," Proc. Nat. Acad. Sci. USA, 1999, 96, 6603-6608.

[11] L. Tsang, J. A. Kong, K.-H. Ding, "Scattering of Electromagnetic Waves: Theories and Applications,” New York: Wiley, 2000.

[12] Italian national research council, "An Internet resource for the calculation of the Dielectric Properties of Body Tissues in the frequency range $10 \mathrm{~Hz}$ - $100 \mathrm{GHz}$, http://niremf.ifac.cnr.it/tissprop/ 